\title{
Offenlegung geldwerter Leistungen durch die Pharmaindustrie
}

\section{Jürg Granwehr}

lic. iur., Rechtsanwalt, Leiter Pharma, scienceindustries

Am 1. Januar 2014 trat der Pharma-Kooperations-Kodex in Kraft. Gemäss diesem legen Pharmaunternehmen Zahlungen an Gesundheitsdienstleister offen. Dies wird 2016 zum ersten Mal der Fall sein, wobei EFPIA sowie scienceindustries den Unterzeichnerfirmen empfehlen, die Daten vom 20. bis zum 30. Juni 2016 offenzulegen.

Bereits in der Ausgabe Nr. 2014/07 der Schweizerischen Ärztezeitung [1] orientierte scienceindustries über die Transparenzinitiative der Pharmaindustrie. Die unmittelbar bevorstehende, erste Publikation der Daten nimmt sie zum Anlass, diese Initiative noch einmal kurz zu erläutern.

\section{Trend zu mehr Transparenz}

Aufgrund einer internationalen Entwicklung zu mehr Transparenz im Verkehr zwischen Pharmaunternehmen und Dienstleistungserbringern im Gesundheits- wesen hat der europäische Verband der pharmazeutischen Industrie (EFPIA) Mitte 2013 den «EFPIA Code on Disclosure of Transfers of Value from Pharmaceutical Companies to Healthcare Professionals and Healthcare

Von der Offenlegungspflicht sind nur wenige Zahlungen ausgenommen.

Organisations» verabschiedet [2]. Beim EFPIA-Disclosure-Code handelt es sich um ein Rahmenregulativ, welches auf Ebene der Länderverbände durch Erlass eigener Kodizes national umgesetzt wurde. Dabei be-

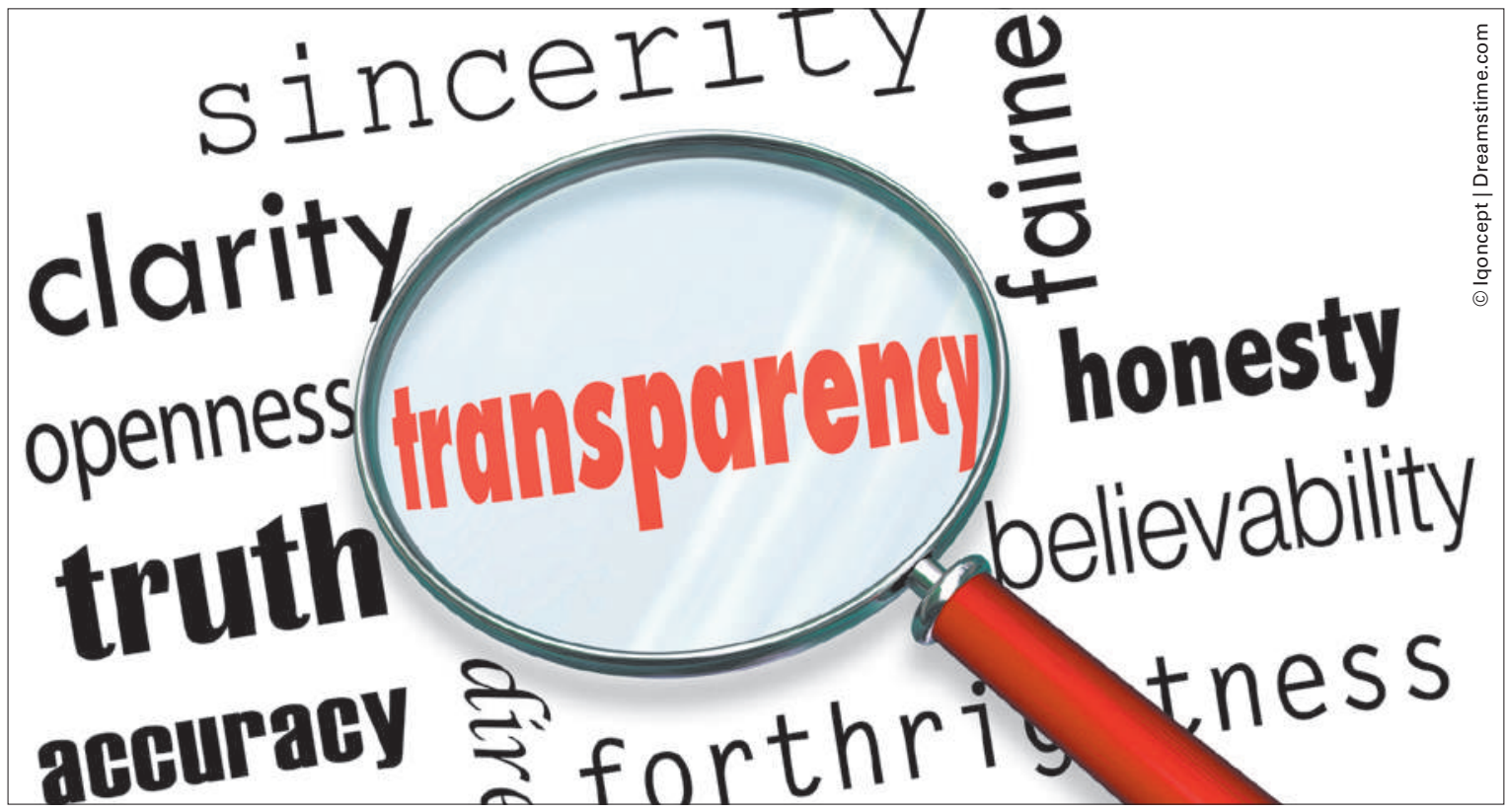

Seit Inkrafttreten des Pharma-Kooperations-Kodex müssen diejenigen Firmen, die diese Erklärung unterzeichnet haben, Zahlungen an Gesundheitsdienstleister offenlegen. Dazu zählen unter anderem Spenden und Zuwendungen an Organisationen, Beiträge an Kosten von Veranstaltungen sowie geldwerte Leistungen für Forschung \& Entwicklung. 
rücksichtigten sie die Rahmenbedingungen der jeweiligen Rechtsordnungen sowie die kulturellen Gegebenheiten und Gewohnheiten.

\section{Umsetzung in der Schweiz}

In der Schweiz ist scienceindustries dafür zuständig. Koordiniert mit ihren Partnerverbänden hat sie den Pharma-Kooperations-Kodex (PKK) ausgearbeitet, welcher per 1. Januar 2014 in Kraft getreten ist [3]. Der PKK regelt die Kooperation der Pharmaunternehmen mit Fachpersonen der Gesundheitsversorgung (Ärzte, Zahnärzte, Apotheker etc.), entsprechenden Organisationen (Spitälern, Forschungsinstitutionen, Fachgesellschaften etc.) und Patientenorganisationen, sowie die Geldflüsse zwischen diesen. Seit Inkrafttreten des PKK müssen Pharmaunternehmen geldwerte Leistungen im Zusammenhang mit verschreibungspflichtigen Arzneimitteln an diese Kooperationspartner offenlegen.

\section{Erste Offenlegung ab dem 20. Juni 2016}

Diese Offenlegungspflicht gilt für jene Firmen, die sich durch Unterzeichnung der entsprechenden Erklärung auf den PKK verpflichtet haben [4]. Sie ist jährlich zu erfüllen, wobei die im Vorjahr geleisteten Zahlungen von den Unternehmen jeweils auf deren Websites publiziert werden. Dies wird 2016 zum ersten Mal der Fall sein, wobei die Verbände den Unterzeichnerfirmen im Sinne einer kohärenten Kommunikation empfehlen, die Daten vom 20. bis zum 30. Juni 2016 offenzulegen.

Korrespondenz:

Jürg Granwehr, lic. iur.

Rechtsanwalt

Leiter Pharma

scienceindustries

Nordstrasse 15

Postfach

CH-8021 Zürich

Tel. +41443681728

juerg.granwehr[at]

scienceindustries.ch

\section{Welche geldwerten Leistungen werden offengelegt?}

Offenzulegen sind Spenden und Zuwendungen an Organisationen, Beiträge an Kosten von Veranstaltungen sowie Entgelte für Dienstleistungen an Organisationen wie Einzelpersonen und schliesslich geldwerte Leis- tungen für Forschung \& Entwicklung. So werden z.B. Honorare für Referate wie auch Beratungsleistungen in Gremien, Beiträge an Anmeldegebühren, aber auch solche an Reise- und Unterkunftskosten im Zusammenhang mit Kongressen oder andern Veranstaltungen transparent ausgewiesen. Von der Offenlegungspflicht sind nur wenige Zahlungen ausgenommen, wie beispielsweise die Abgeltungen für Fachpersonen bei Bestellungen und Lieferungen von Arzneimitteln, die

Ziel der Offenlegungsinitiative ist es, eine weitgehend individuelle Transparenz zu schaffen.

unentgeltliche Abgabe von Mustern verschreibungspflichtiger Arzneimittel oder die Bezahlung von Mahlzeiten bis zu einem maximal zulässigen Betrag von 150.- CHF pro Person und Mahlzeit.

\section{Pflicht zur Offenlegung}

Die Unternehmen weisen die Gesundheitsdienstleistungserbringer beim Vertragsabschluss auf ihre Pflicht hin, die vereinbarten Abgeltungen offenzulegen. Aus datenschutzrechtlichen Gründen müssen die Empfänger der Abgeltung der Offenlegung zustimmen. Ziel der Offenlegungsinitiative ist es, eine weitgehend individuelle Transparenz zu schaffen. Lehnt eine Fachperson oder Gesundheitsversorgungsorganisation die Offenlegung ab, gilt entsprechend die Empfehlung seitens der Verbände an die Pharmaunternehmen, vom Vertragsabschluss abzusehen. Der jeweilige Entscheid liegt bei jedem Unternehmen selbst.
Literatur

1 http://www.saez.ch/archiv/details/neue-verhaltensregeln-fuerdie-pharmaunternehmen-mit-auswirkungen-auf-die-aerzteschaft.html

2 http://transparency.efpia.eu/the-efpia-code-2

3 https://www.scienceindustries.ch/pharmakodizes

4 https://www.scienceindustries.ch/engagements/pharmakodex/ unterzeichner-des-pharma-kooperations-kodexes 